\title{
Approaches to sensitizing glioblastoma to radiotherapy: Use of lentiviral vectors
}

\author{
TEONG LIP CHUAH ${ }^{1,2}$, DAVID GREGORY WALKER ${ }^{1-3}$, MING WEI $^{4}$, \\ SHAUN SCOTT ${ }^{2}$ and MARTIN FRANCIS LAVIN ${ }^{1,2,5}$
}

\author{
${ }^{1}$ Department of Surgery, University of Queensland, St. Lucia; ${ }^{2}$ Radiation Biology and Oncology Unit, Queensland \\ Institute of Medical Research, Herston; ${ }^{3}$ Brizbrain and Spine Neurosurgery and Spine Surgery, Auchenflower; \\ ${ }^{4}$ Gene Therapy Unit, Prince Charles Hospital, Chermside; ${ }^{5}$ University of Queensland \\ Centre for Clinical Research, Herston, Queensland, Australia
}

Received September 19, 2011; Accepted November 7, 2011

DOI: $10.3892 /$ ijo.2012.1409

\begin{abstract}
Glioblastoma multiforme (GBM) is the most common primary brain tumour and extirpation followed by radio- and chemotherapy has had minimal impact on the median survival of patients which is still less than one year. Hence, a novel therapeutic modality is required if the survival of patients with this disease is to be improved. ATM, mutated in the human genetic disorder ataxia-telangiectasia (A-T), plays a central role in the response to DNA double strand breaks and patients with this disorder are characterised by extreme sensitivity to radiation, increased risk of cancer and neurodegeneration. Thus, ATM represents a potential target for radiosensitization of brain tumour cells. A safe, non-replicating lentivirus is used to abrogate ATM in GBM through the antisense and RNAi approaches for radiosensitization. With either techniques, ATM protein was reduced by $>90 \%$ and there was a 3-fold sensitization of GBM cells to radiation. ATM protein activation as well as ATM pS1981 foci formation were defective and downstream signalling determined by Ser15 phosphorylation on p53 was reduced. Success in the approaches provides a novel and exciting strategy for the treatment of GBM and thus improving the survival of patients with these tumours.
\end{abstract}

\section{Introduction}

Glioblastoma multiforme (GBM) is the most common intraparenchymal brain tumour and remains a neurosurgical conundrum whereby patients usually succumb to the disease

Correspondence to: Dr Martin F. Lavin, Radiation Biology and Oncology Unit, Queensland Institute of Medical Research, 300 Herston Road, Herston, QLD 4006, Australia

E-mail: martin.lavin@qimr.edu.au

Key words: radiosensitization, glioblastoma multiforme, RNAi, antisense, ataxia-telangiectasia mutated, lentivirus within one year. Surgery followed by radiotherapy and chemotherapy have had minimal impact on the prognosis of patients over the years, although with this combination, the median survival of patients with GBM is increased from 4 months (with surgery alone) to 9 months. However, it is not possible to increase the current recommended radiation dose of 45-60 Gy to achieve greater tumour control as the side effects of radiotherapy will increase. Hence, novel therapeutic modalities are essential to improve the survival of patients diagnosed with this oncological enigma.

The ATM (ataxia-telangiectasia mutated) gene spans approximately $150 \mathrm{~kb}$ of genomic DNA and consists of 66 exons (1). ATM is critical in the activation of cell cycle checkpoints in the presence of DNA double strand breaks induced by ionizing radiation (2). Mutations of the ATM gene lead to the autosomal recessive disease ataxia-telangiectasia (A-T) with cellular phenotypes including sensitivity to ionizing radiation, defects at G1/S and G2/M cell-cycle checkpoint, chromosomal instability and increased chromosomal breakages (3). These features are well-demonstrated in previously-published papers and sensitization to ionizing radiation is imposed on cells with reduced ATM protein expression (4,5).

The results of gene therapy clinical trials for patients with malignant gliomas have been disappointing since only a small proportion (5-15\%) of GBM tumour cells are in an active growth phase. Poor clinical results are attributed to the retrovirus inability to transduce non-dividing cells (6) and the strategy of radiosensitizing U87 cells through antisense ATM cDNA cloned into a retroviral vector (7) may again produce disappointing clinical outcomes. Adenovirus-mediated antisense ATM gene transfer had been used to radiosensitize prostate cancer cells (8) but the immunogenic response against adenoviruses prevents the safe use of this vector. To overcome these concerns, a lentiviral vector was utilized here as it could enter the nucleus of quiescent cells by active transportation through the nuclear pore import mechanism (9) and thus ensured the efficient transduction of non-mitotic GBM cells.

Hence, to achieve greater GBM tumour control at the current or lower radiation doses, we postulated that GBM 
tumour cells would be sensitized to radiation treatment by reducing the expression of ATM through either antisense or RNAi gene therapy delivered by lentiviruses. The reduction in expression of ATM using both methodologies led to disruption of ATM signalling pathways and the transduced cells were sensitized to radiation.

\section{Materials and methods}

Construction of the pHRCSCMVatsATM2.3kb(antisense ATM) and pHRCSCMV (control) lentiviral vectors. The lentiviral plasmids were provided as kind gifts from the Gene Therapy Unit, Prince Charles Hospital. Three lentiviruses were packaged: pHRCMVGFP, pHRCSCMV and pHRCSCMVatsATM2.3kb. A $2.3-\mathrm{kb}$ fragment containing the $7442-9746 \mathrm{~kb}$ region of the ATM cDNA from the pEAT1-1 vector was cloned in the antisense direction into the XhoI-Bsu36I site of the pHRCS (lentiviral transfer) vector. Transient packaging of lentiviruses were conducted using 293T cells with the calcium phosphate precipitation method. pHRCMVGFP lentiviruses were used to titer the lentiviral concentrations and this methodology was previously described (10).

Construction of the pLLRNAIATM and pLLSCATM lentiviruses. The Lentilox RNAi lentiviral vector or pLL3.7 was provided as a kind gift from the Centre for Cancer Research, Massachusetts Institute of Technology (11). Three lentiviruses were packaged: pLL (pLL3.7), pLLRNAiATM and pLLScATM. Chemically synthesised oligonucleotides, which contained either RNAi ATM (RNAiATM) (Fig. 1A) or scrambled ATM (ScATM) (Fig. 1B) sequences, were ordered from Sigma Genosys Australia Pty Ltd. The 55 and 59 bp oligonucleotides were annealed to generate double-stranded DNA with an XhoI overhang and cloned into the HpaI-XhoI site of the pLL3.7 lentiviral vector. The methodology for cloning of shRNAs into pLL3.7 vector was previously described (10). To ensure the correct clones were obtained, the pLLRNAiATM and pLLScATM vectors were sequenced in both the forward and reverse directions. Sequence of the forward primer was: GTTTATTACAGGGACAGCAGAG and sequence of the reverse primer was: TGGCGGTAATACGGTTATC. Transient packaging of lentiviruses was conducted using 293T cells with the Lipofectamine ${ }^{\mathrm{TM}} 2000$ (Invitrogen) reagent and the methodology for lentivirus titering was previously described (10).

Cell culture. 293T (human embryonic kidney cells with SV40 large T antigen), U118 (human glioblastoma multiforme cells), AT5BIVA (SV40-transformed fibroblast cell line from a patient with A-T), C3ABR and AT25ABR cells were cultured with RPMI-1640 (Gibco) with 7\% heat inactivated foetal calf serum (FCS) and $1 \%$ penicillin/streptomycin $(\mathrm{P} / \mathrm{S})$.

Transduction of U118 cells. U118 cells were transduced at MOI (multiplicity of infection) 5 for the antisense ATM experiments using the pHRCSCMV and pHRCSCMVatsATM2.3kb lentiviruses, and MOI 2 for the RNAi ATM experiments using the pLL (pLL3.7), pLLRNAiATM and pLLScATM lentiviruses. FACS analysis was conducted on these cells and transduced cells with high EGFP expression were sorted using a MoFlo (DakoCytomation, USA).
$\mathbf{A}$

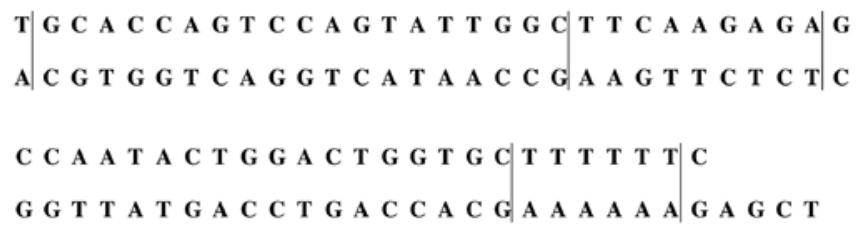

B

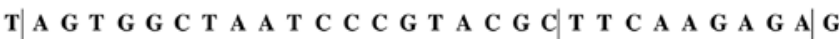

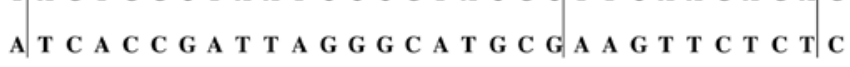

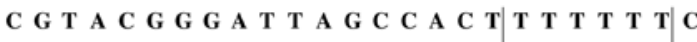

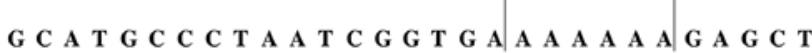

Figure 1. Sequences of the RNAi ATM (RNAiATM) and scrambled ATM (ScATM) oligonucleotides. (A) Sequences of the RNAiATM oligonucleotides. (B) Sequences of the ScATM oligonucleotides.

Radiation procedure. The cells were irradiated with gammarays using a ${ }^{137} \mathrm{Cs}$ Gammacell ${ }^{\circledR} 40$ Exactor irradiator (MDS Nordion, Ontario, Canada) at 6 or 10 Gy.

Protein extraction and immunoblotting. Nuclear protein was extracted from unirradiated, 1 h post- 6 Gy and post-10 Gy of irradiation of non-transduced and transduced U118 cells. Total protein was extracted from the C3ABR and AT25ABR cell pellets. The methodology for protein extraction was previously described (4). Immunoblotting was performed using the standard protocol (12). The filters were probed with the ATM-2c1 monoclonal antibody (GeneTex, USA) for ATM, with the ATM Protein Kinase S1981 polyclonal antibody (Rockland, USA) for phospho-S1981 ATM, with the Ab-2 monoclonal antibody (Calbiochem, USA) for DNA-PK, with the phospho-p53 (Ser 15) polyclonal antibody (Cell Signaling Technology, Inc., USA) for phospho-S15 p53, with the Pab 1801 monoclonal antibody (Santa Cruz Biotechnology, USA) for p53 and with the AC-40 monoclonal antibody (Sigma, USA) for actin. The immunoblots were scanned using a laser densitometer and analysis of band intensities was conducted using the ImageQuant ${ }^{\circledR}$ software with individual band intensities normalised to the control band for each sample. Immunoblotting was repeated to ensure reproducibility.

Immunostaining of cells. U118 cells transduced by pLL, pLLRNAiATM and pLLScATM lentiviruses were grown on glass coverslips. They were irradiated with $10 \mathrm{~Gy}$ and fixed after $15 \mathrm{~min}$ post-irradiation. The cells were probed with ATM Protein Kinase S1981 polyclonal antibody (Rockland) and incubated with anti-rabbit immunoglobulin $\mathrm{G}$ antibody conjugated to Alexa Fluor ${ }^{\circledR} 594$ dye (Invitrogen, USA). The protocol for immunostaining was previously described (13). At least two hundred cells were counted for each group and the entire experiment was independently repeated.

Clonogenic assay. Control and ATM knockdown U118 cells were plated in triplicates in $10-\mathrm{cm}$ polystyrene tissue culture 
A

B

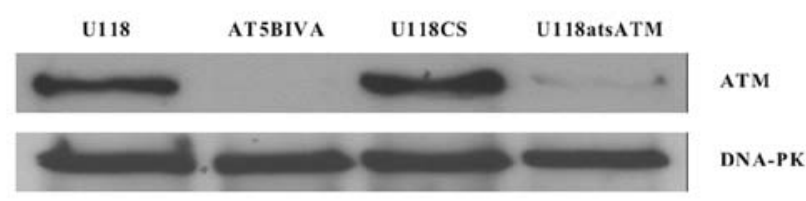

C

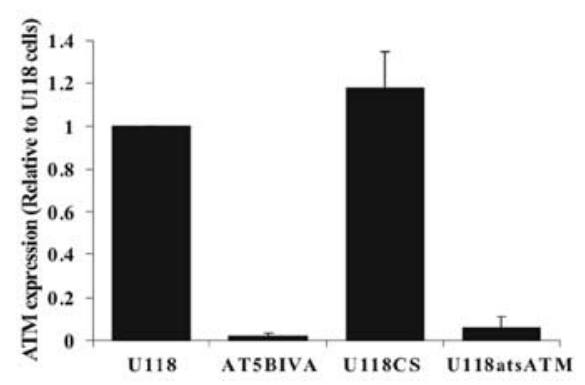

Figure 2. Down-regulation of ATM in U118 cells using antisense ATM lentiviruses. (A) Schematic diagram of the pHRCS lentiviral transfer vector. The lentiviral vector contains a multiple cloning site (MCS) located downstream from the CMV promoter. Other sequences in this vector include 5' long terminal repeat (5'-LTR), splice donor site (SD), HIV packaging signal ( $\Psi)$, truncated gag, REV response element (RRE), woodchuck post-regulatory response element (WPRE) and 3' long terminal repeat with partial U3 deletion (3'-LTR). (B) Analysis of ATM protein and DNAPK expression by immunoblotting of nuclear extracts from U118 cells, AT5BIVA cells, U118 cells transduced by the pHRCSCMV lentiviruses (U118CS) and U118 cells transduced by the pHRCSCMVatsATM2.3kb (U118atsATM) lentiviruses Analysis of DNAPK protein from the same immunoblot verifies that loading is equal. (C) The percentage of ATM protein expression of the various cells compared to the non-transduced U118 cells is represented by this graph and the values are expressed as mean \pm SD of three separate experiments.

plates (TPP) as following: 2000 cells (for no irradiation and irradiation at $1 \mathrm{~Gy}$ ), 4000 cells (for irradiation at $2 \mathrm{~Gy}$ ), 8000 cells (for irradiation at $4 \mathrm{~Gy}$ ) and 16000 (for irradiation at $6 \mathrm{~Gy})$. The protocol for this assay was previously described (14). The entire experiment was independently repeated and the clonogenic ability of the ATM knockdown U118 cells and controls were analysed, survival fractions were calculated and normalised to the plating efficiencies.

\section{Results}

Attenuation of ATM protein expression and radiosensitization using antisense ATM lentiviruses. The pHRCSCMVatsATM $2.3 \mathrm{~kb}$ vector was generated using the pHRCS plasmid (Fig. 2A). The sense fragment of the 7442-9746 kb region of the ATM cDNA was not used as negative control as it had been demonstrated that a truncated PI-3K domain of the ATM protein could have a radioprotective effect on target cells (15). Bands, which migrated in the expected size for the ATM protein $(350 \mathrm{kDa})$, were revealed in approximately equal amounts with nuclear extracts from the U118 cells and
$\mathbf{A}$

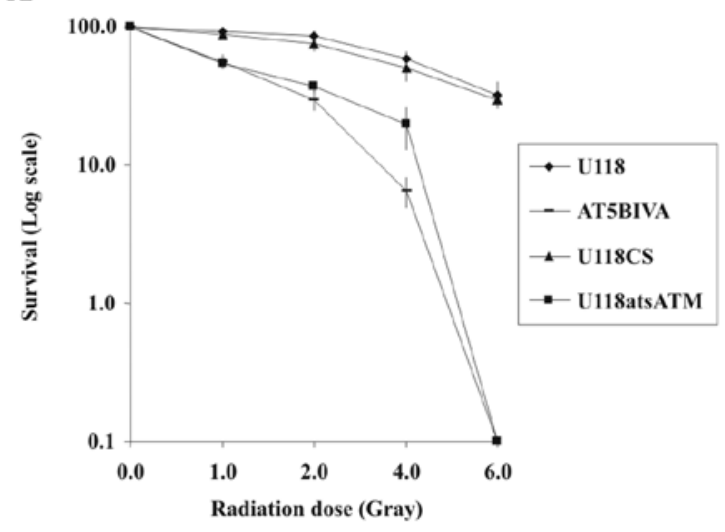

B

$\begin{array}{lllll} & \text { U118 } & \text { U118CS } & \text { U118atsATM } & \text { AT5BIVA } \\ \text { SF2 (A) } & 79.3 & 76.7 & 35.3 & 33.6 \\ \text { SF2 (B) } & 90.1 & 65.6 & 41.2 & 24.6 \\ \text { SF2 (C) } & 89.2 & 83.3 & 35.3 & 31.1 \\ \text { Mean SF2 } & 0.86 & 0.75 & 0.37 & 0.30 \\ \text { Standard Deviation } & 0.06 & 0.089 & 0.034 & 0.046\end{array}$

Figure 3. Radiosensitization of U118 cells using antisense ATM lentiviruses. (A) Clonogenic survival curves of U118 cells, AT5BIVA cells, U118 cells transduced by the pHRCSCMV lentiviruses (U118CS) and U118 cells transduced by the pHRCSCMVatsATM2.3kb lentiviruses (U118atsATM). (B) The survival fractions after 2 Gy irradiation (SF2) of U118 cells, AT5BIVA cells, U118 cells transduced by the pHRCSCMV lentiviruses (U118CS) and U118 cells transduced by the pHRCSCMVatsATM2.3kb lentiviruses (U118atsATM). This entire experiment was performed in triplicates and independently conducted to a total of three times.

U118 cells transduced by the pHRCSCMV (control) lentiviruses (Fig. 2B). The AT5BIVA cell line was previously generated from a homozygous A-T patient and as demonstrated, there was no ATM protein expression in this cell line. ATM protein expression was demonstrated to be significantly reduced in U118 cells transduced by the pHRCSCMVatsATM2.3kb (antisense ATM) lentiviruses (Fig. 2B and C).

The survival curves in Fig. 3A demonstrate that U118 cells transduced by pHRCSCMVatsATM2.3kb (antisense ATM) lentiviruses were sensitized to ionizing radiation and transduction by pHRCSCMV (control) lentiviruses did not affect the survival of U118 cells after irradiation. The most commonly used external beam radiotherapy fractionated daily dosage for the glioblastoma multiforme is $2 \mathrm{~Gy}$. The survival fractions after 2 Gy irradiation (SF2) of U118 cells transduced by the pHRCSCMVatsATM2.3kb lentiviruses (0.37) was significantly lower $(\mathrm{P}=0.0001)$ than the U118 cells $(0.86)$ and U118 cells transduced by the pHRCSCMV lentiviruses (0.75) by Student's t-test (Fig. 3B). The survival fraction of the AT5BIVA cells was 0.30 . This demonstrates a significant increase in radiosensitivity of the U118 cells transduced by the pHRCSCMVatsATM2.3kb lentiviruses.

Attenuation of ATM protein expression by RNAi methodology. Transduction of U118 cells by pLLRNAiATM lentiviruses led to down-regulation of ATM protein expression (Fig. 4A). 
A

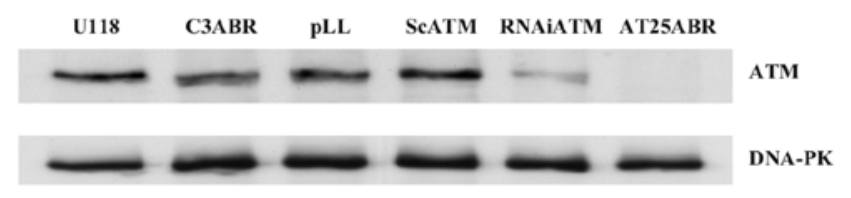

B

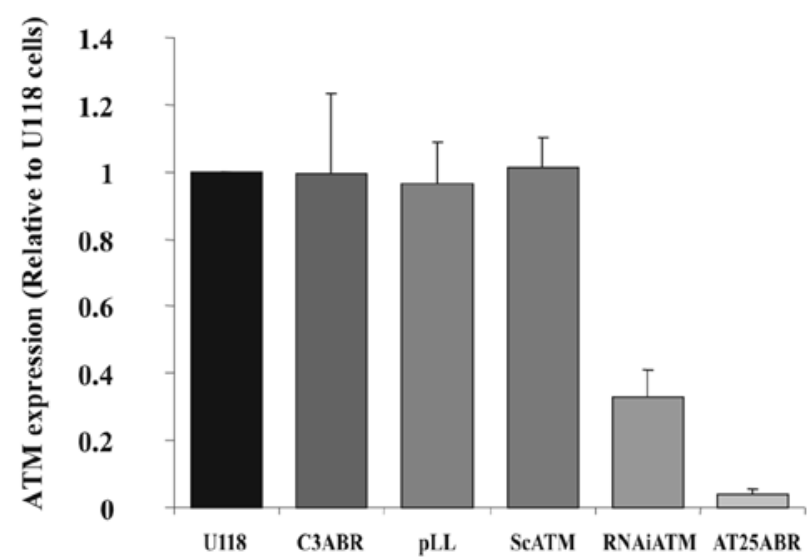

Figure 4. Down-regulation of ATM in U118 cells using pLLRNAiATM lentiviruses. (A) Immunoblots of ATM and DNAPK with nuclear extracts from U118 cells (U118), C3ABR cells, U118 cells transduced by the pLL lentiviruses (pLL), U118 cells transduced by the pLLScATM lentiviruses (ScATM), U118 cells transduced with pLLRNAiATM lentiviruses (RNAiATM) and AT25ABR cells. Analysis of DNAPK protein from the same immunoblot verifies that loading is equal. (B) This graph demonstrates the percentage of ATM protein expression of the various cells compared to the non-transduced U118 cells and the values are expressed as mean \pm SD of three separate experiments.

C3ABR and AT25ABR cells, non-transduced U118 cells, U118 cells transduced by the pLL and pLLScATM lentiviruses were used as controls. As demonstrated in Fig. 4, the control (pLL and pLLScATM) lentiviruses did not affect ATM protein expression in the transduced U118 cells as ATM protein levels in these cells were comparable to the non-transduced U118 and C3ABR cells. For reference, AT25ABR cells were utilized to demonstrate that expression of ATM protein was absent in a homozygous A-T cell line.

\section{Effect of ATM RNAi on DNA damage signalling}

Reduced S1981 ATM autophosphorylation. Exposure of cells to ionizing radiation leads to prompt autophosphorylation of ATM kinase on S1981 which activates the enzyme by dissociation of the inactive ATM dimer into an active monomer $(16,17)$. Since ATM protein was reduced by pLLRNAiATM lentiviruses, it was anticipated that its activation would also be affected. The results in Fig. 5A demonstrate that whilst radiation-induced phosphorylation of S1981 was observed in non-transduced U118 cells and U118 cells transduced by the pLL and pLLScATM lentiviruses, U118 cells transduced by the pLLRNAiATM lentiviruses had no detectable S1981 phosphorylation.

Reduced S15 p53 phosphorylation. Cell cycle checkpoints are defective in A-T cells (18) including p53 activation by ATM $(19,20)$. In the absence of ionizing radiation, there was minimal
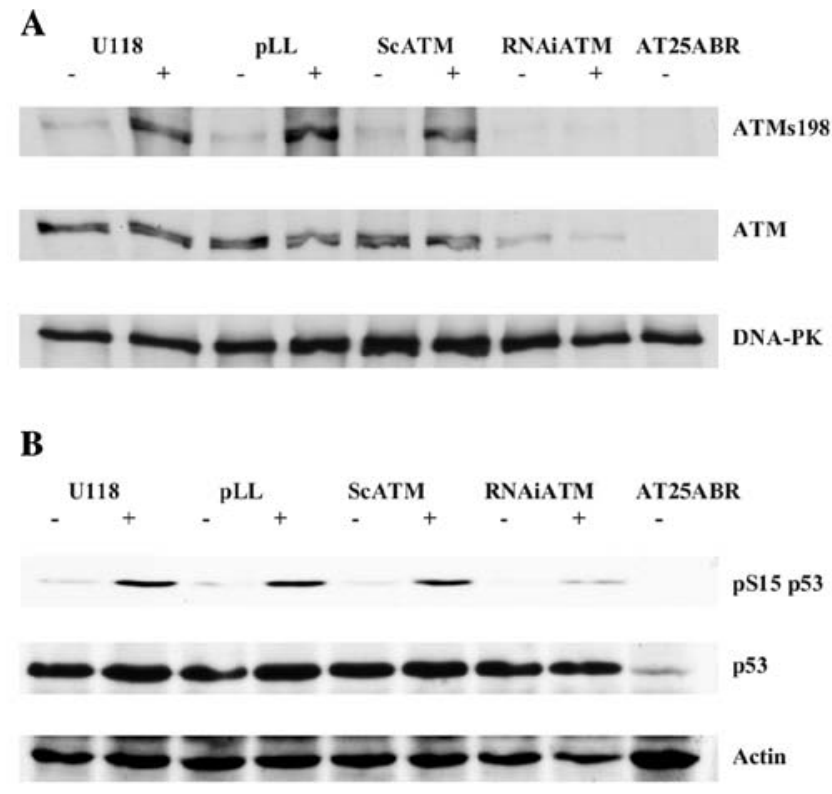

Figure 5. S1981 ATM autophosphorylation and S15 p53 phosphorylation in U118 cells transduced by pLLRNAiATM lentiviruses after exposure of cells to ionizing radiation. (A) Negligible S1981 ATM autophosphorylation in U118 cells transduced by pLLRNAiATM lentiviruses after exposure of cells to ionizing radiation. Immunoblots of ATM-s1981, ATM and DNAPK with nuclear extracts from unirradiated (-) and $1 \mathrm{~h}$ post-10 Gy irradiated (+) U118 cells (U118), U118 cells transduced by the pLL lentiviruses (pLL), U118 cells transduced by the pLLScATM lentiviruses (ScATM), U118 cells transduced with pLLRNAiATM lentiviruses (RNAiATM) and AT25ABR cells. Immunoblots are a representation of two separate experiments. (B) Reduced S15 p53 phosphorylation in U118 cells transduced by pLLRNAiATM lentiviruses after exposure of cells to ionizing radiation. Immunoblots of pS15 p53, p53 and actin with nuclear extracts from unirradiated (-) and $1 \mathrm{~h}$ post- 6 Gy irradiated (+) U118 cells (U118), U118 cells transduced by the pLL lentiviruses (pLL), U118 cells transduced by the pLLScATM lentiviruses (ScATM), U118 cells transduced with pLLRNAiATM lentiviruses (RNAiATM) and AT25ABR cells. Immunoblots are a representation of three separate experiments.

detection of pS15 p53 in the unirradiated cells (Fig. 5B). After 6-Gy ionizing radiation exposure, $\mathrm{p} 53$ phosphorylation on $\mathrm{S} 15$ at 60 min was induced in the U118 cells transduced by pLL and pLLScATM lentiviruses similar to the level observed in the non-transduced U118 cells. On the contrary, an attenuated p53 response to radiation exposure was evident in U118 cells transduced by pLLRNAiATM lentiviruses. This demonstrates that attenuation of ATM protein expression resulted in reduced downstream signalling such as p53 phosphorylation in these cells.

Reduced phospho-S1981 ATM foci formation. Exposure to ionizing radiation produces rapid recruitment of DNA damage recognition proteins as small granular foci to the sites of DNA damage (21). It was demonstrated that immunofluorescent staining of phospho-S1981 ATM was diffuse across the nucleus in fibroblasts at the earliest time-point after irradiation and discrete pS1981 ATM foci were evident after several minutes (16). As ATM protein expression was attenuated in U118 cells transduced by pLLRNAiATM lentiviruses, the formation of discrete pS1981 ATM foci in U118 cells was investigated 15 min after exposure to 10 Gy irradiation. pS1981 ATM foci were detected in the majority of U118 cells transduced by the 


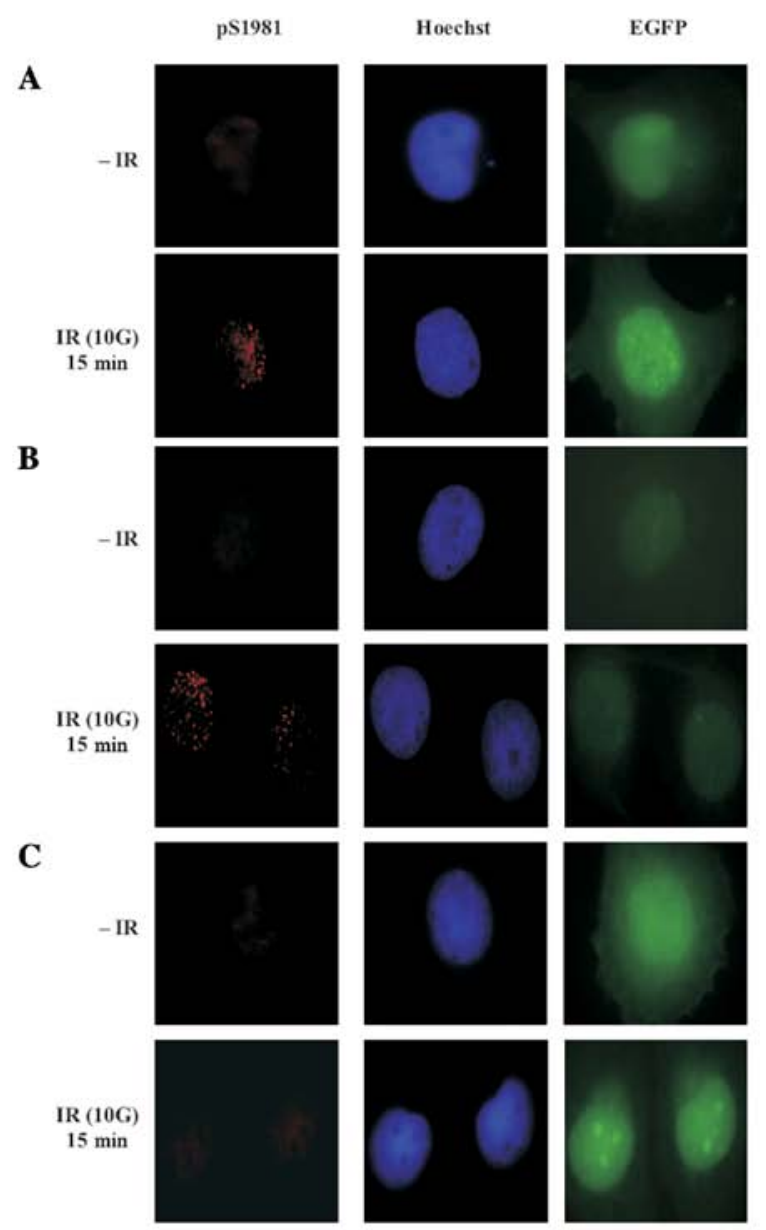

D

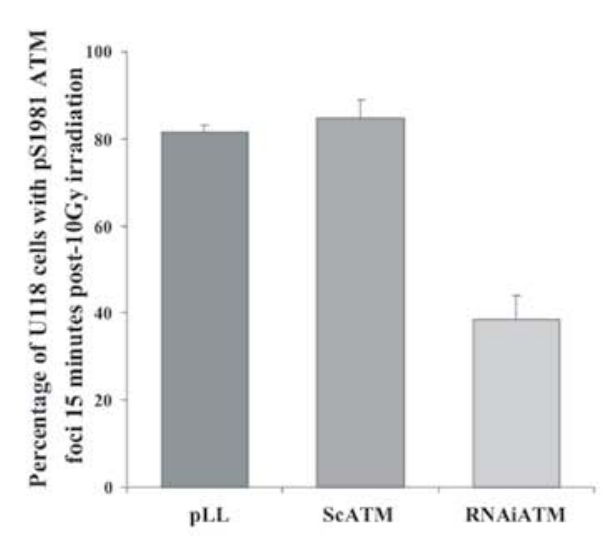

Figure 6. Reduced phospho-S1981 ATM foci formation in U118 cells transduced by pLLRNAiATM lentiviruses after exposure to ionizing radiation. (A) pS1981 ATM foci formation in U118 cells transduced by pLL lentiviruses. (B) pS1981 ATM foci formation in U118 cells transduced by pLLScATM lentiviruses. (C) Reduced pS1981 ATM foci formation in U118 cells transduced by pLLRNAiATM lentiviruses. Hoechst 33342 (Sigma) was used as nuclear stain and all transduced U118 cells had EGFP expression. (D) This graph demonstrate the percentage of U118 cells transduced by pLL and pLLScATM lentiviruses compared to U118 cells transduced by pLLRNAiATM lentiviruses that have pS1981 ATM foci 15 min post-10 Gy irradiation. At least two hundred cells were counted for each column of the graph and the values are expressed as mean \pm SD of two separate experiments.

control lentiviruses pLL (Fig. 6A) and pLLScATM (Fig. 6B) after exposure to ionizing radiation. In contrast, the number of U118 cells transduced by pLLRNAiATM lentiviruses
$\mathbf{A}$

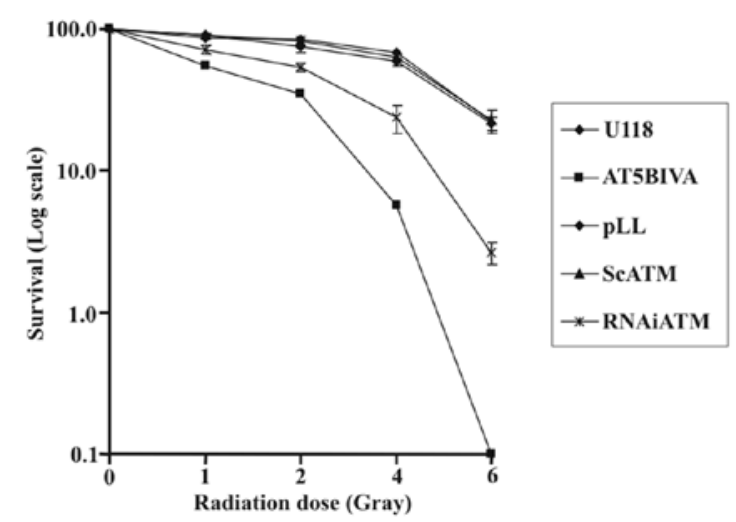

B

$\begin{array}{llllll} & \text { U118 } & \text { pLL } & \text { ScATM } & \text { RNAiATM } & \text { AT5BIVA } \\ \text { SF2 (A) } & 86.1 & 69.2 & 86.8 & 50 & 36.6 \\ \text { SF2 (B) } & 84.2 & 82.4 & 76.7 & 54.6 & 35.1 \\ \text { SF2 (C) } & 83.7 & 73.1 & 85.2 & 57.6 & 33.4 \\ \text { Mean SF2 } & 0.85 & 0.75 & 0.83 & 0.54 & 0.35 \\ \text { Standard Deviation } & 0.013 & 0.068 & 0.054 & 0.038 & 0.016\end{array}$

Figure 7. Radiosensitization of U118 cells using pLLRNAiATM lentiviruses. (A) Clonogenic survival curves and (B) survival fractions after 2 Gy irradiation (SF2) of U118 cells (U118), U118 cells transduced by the pLL lentiviruses (pLL), U118 cells transduced by the pLLScATM lentiviruses (ScATM), U118 cells transduced with pLLRNAiATM lentiviruses (RNAiATM) and AT5BIVA cells. The entire experiment was conducted in triplicates and independently repeated.

displaying pS1981 ATM foci after irradiation was reduced (Fig. 6C). As demonstrated in Fig. 6D, the presence of pS1981 ATM foci was detected at $15 \mathrm{~min}$ after $10 \mathrm{~Gy}$ irradiation in the majority of U118 cells transduced by pLL (81.6\%) and pLLScATM lentiviruses (84.6\%). In contrast, the proportion of U118 cells transduced by pLLRNAiATM lentiviruses exhibiting pS1981 ATM foci at 15 min after 10 Gy irradiation was considerably diminished $(38.4 \%)$. This demonstrates that the response to DNA damage in U118 cells with reduced ATM protein expression is significantly impaired.

Radiosensitivity. As ATM protein expression was reduced in U118 cells transduced with pLLRNAiATM lentiviruses, the survival of these cells after exposure to ionizing radiation was examined by clonogenic assays to determine whether a radiosensitive phenotype resulted from the transduction with pLLRNAiATM lentiviruses. Clonogenic survival curves of U118 cells, AT5BIVA cells, U118 cells transduced by the pLL, pLLScATM and pLLRNAiATM lentiviruses were generated (Fig. 7A). These data demonstrate that U118 cells transduced by pLLRNAiATM lentiviruses have been sensitized to ionizing radiation when compared to the non-transduced U118 cells and U118 cells transduced by the control (pLL and pLLScATM) lentiviruses. Adjuvant radiotherapy for GBM involves a total radiation dose of 45-60 Gy with a maximal daily dose of $2 \mathrm{~Gy}$. With this in mind, the survival fraction of nontransduced and transduced U118 cells after 2 Gy irradiation 
(SF2) was examined. The SF2 of U118 cells transduced by the pLLRNAiATM lentiviruses (0.54) was reduced compared to non-transduced U118 cells ( 0.85$)$ and U118 cells transduced by the pLL (0.75) and pLLScATM (0.83) lentiviruses $(\mathrm{P}<0.0001$; Student's t-test) (Fig. 7B).

\section{Discussion}

Malignant glioma is one of the most intractable diseases in humans (22) with a median survival of less than one year (23). It is well known that a combination of surgery and radiotherapy doubles the median survival of patients to about 9 months. The approach of increasing the current dose of radiotherapy from 45-60 Gy to higher dosages may eradicate more tumour cells but is likely to result in radiation-induced destruction of adjacent neuronal tissues. There are currently more than 40 clinical gene therapy trials on malignant gliomas which generally involve the direct injection of suicide, cytokine or tumour suppressor gene transfer vectors into the margin of the surgical cavity or tumour bulk intraoperatively (6). Although these novel therapeutic modalities have been demonstrated to be safe and well-tolerated, clinical efficacy has been unsatisfactory due to poor transduction of target malignant cells.

Both the antisense and RNAi ATM lentiviruses target the ATM signal transduction pathway of the radioresistant GBM cells to increase the efficacy of radiotherapy. In contrast to previous trials to improve radiation treatment by pharmacological radiosensitizers, the proposed method of radiosensitization intends to radiosensitize all tumour cells sharing a common genetic feature. In 2000, a similar strategy to this research work was published whereby antisense ATM cDNA was cloned into a retroviral vector and transfected into U87 cells which was selected in the presence of genticin (7). As neurons are generally quiescent, retroviral vectors were used in many gene therapy trials of GBM based on their property of transducing dividing cells only (6). However, the usefulness of retroviruses is limited by low production titers, instability of the virion and the inability to transduce non-dividing cells (24). Furthermore, tumour kinetics of the GBM demonstrated that only a small proportion (5-15\%) of tumour cells are in active growth phase and hence, the efficacy of cell cyclespecific therapeutic agents will be reduced. To target quiescent tumour cells, adenoviruses can be used and radiosensitization of prostate cancer cells using adenovirus-mediated antisense ATM gene transfer was published in 2000 (8). However, the host's immune response against the adenovirus is a major obstacle to the safe and effective use of this vector (9) and it has been shown that potent cellular immune response to adenovirus-infected cells does not require de novo viral gene expression (25). To overcome these concerns, lentiviruses were used in this study for ATM knockdown gene transfer as they are able to transduce quiescent cells (9) and have low immunogenicity (26).

This paper demonstrates that the expression of the ATM gene in the radioresistant glioblastoma multiforme cells can be effectively down-regulated by lentiviruses expressing antisense ATM or shRNAs to the ATM gene as substantiated by the reduction in the ATM protein level in these cells. In contrast, ATM protein expression in U118 cells infected by control lentiviruses remained unchanged. Radiation-induced phosphorylation on S1981 was absent in U118 cells transduced by the pLLRNAiATM lentiviruses and discrete pS1981 ATM foci formation was absent in the majority of these cells. Defective activation of ATM with abrogation of downstream signalling is well-characterised (4,5). After exposure to ionizing radiation, Mre11-Rad50-NBS1 (MRN) complex is recruited to the site of double-strand break and ATM is recruited to the flanking regions of the break whereby it is partially activated $(27,28)$. MRN complex then recruits ATM to the site of double-strand break (29) and the inactive ATM dimer is dissociated into an activated monomer (16) and autophosphorylated on S367, S1893 and S1981 (17). ATM activation leads to MRN complex phosphorylation (29) and cell-cycle checkpoints activation $(30,31)$. The immunobloting and immunostaining results for phospho-S1981 ATM support the fact that the response to DNA damage in U118 cells with reduced ATM protein expression is significantly impaired and the migration of the activated ATM protein to the sites of DNA damage is abated.

p53 is critical for the protection of genome integrity and with a variety of stimuli, this tumour suppressor gene is rapidly stabilised and activated. When activated, p53 induces cell cycle arrest by p21 transactivation or induction of apoptosis by both transcription-dependent and independent pathways $(32,33)$. One of the significant characteristics in cells with down-regulation of the ATM gene expression is the failure of p53 stabilisation (4). Defective S15 p53 phosphorylation and p53 stabilisation in the presence of DNA double-strand breaks is a prominent attribute of the disease ataxia-telangiectasia (34). The results in this study demonstrated that p53 response to radiation exposure was attenuated in U118 cells transduced by pLLRNAiATM lentiviruses in contrast to U118 cells transduced by control lentiviruses. These results support the fact that reduced ATM protein expression and activation in the U118 cells results in impaired p53 signalling. The intrinsic radiosensitivity of human tumours can be quantitatively expressed as the survival fraction after treatment with a clinically relevant radiation dose of $2 \mathrm{~Gy}$ (SF2) (8). The results of the clonogenic assays demonstrate that the SF2 of U118 cells transduced by the pHRCSCMVatsATM2.3kb or pLLRNAiATM lentiviruses was reduced compared to non-transduced U118 cells and U118 cells transduced by control lentiviruses. The reduction in the survival fraction of the glioblastoma multiforme cells transduced by pHRCSCMVatsATM2.3kb or pLLRNAiATM lentiviruses after treatment with 2 Gy radiation dose demonstrates radiosensitization of these transduced cells.

The use of lentiviral vectors will improve transduction efficiencies and immunological side effects caused by adenoviruses can be avoided. The advantages of the lentiviral vector include vector insert capacities up to $10 \mathrm{~kb}$, long-term transgene expression in transduced cells, high titre viral production and low immunogenicity (26). Furthermore, as only $5-15 \%$ of GBM tumour cells are in active growth, the lentiviral vector will ensure efficient transduction of these cells due to its ability to enter the nucleus of quiescent cells by active transportation through the nuclear pore import mechanism (9). Alternative approaches have been used to target ATM to sensitize cells to radiation $(35,36)$. However, the concentration of KU-55933 used for effective inhibition $(10 \mu \mathrm{M})$ is too high to be useful in a therapeutic setting and clearly it is desirable to generate addi- 
tional specific inhibitors of ATM with greater efficacy. ATM knockdown lentiviruses can be administered intraoperatively to residual GBM tumour cells followed by conventional postoperative external beam radiation therapy. With this strategy, localised eradication of remaining GBM tumour cells can be achieved with minimal damage to the surrounding healthy brain and tumour control can be effectively accomplished at lower radiation doses. Moreover, if the current radiation dose is utilized in the treatment of GBM with reduced ATM gene expression, more tumour cells can be eradicated. Sustained effort in the development of new strategies for the treatment of glioblastoma multiforme, such as the one proposed in this paper, may one day allow significant improvement in the survival of patients with this cancer and perhaps even a cure for this disease.

\section{References}

1. Uziel T, Savitsky K, Platzer M, et al: Genomic Organization of the ATM gene. Genomics 33: 317-320, 1996.

2. Lavin MF and Kozlov S: ATM activation and DNA damage response. Cell Cycle 6: 931-942, 2007.

3. Kastan MB and Lim DS: The many substrates and functions of ATM. Nat Rev Mol Cell Biol 1: 179-186, 2000.

4. Zhang N, Chen P, Gatei M, Scott S, Khanna KK and Lavin MF: An anti-sense construct of full-length ATM cDNA imposes a radiosensitive phenotype on normal cells. Oncogene 17: 811-818, 1998.

5. Uhrhammer N, Fritz E, Boyden L and Meyn MS: Human fibroblasts transfected with an ATM antisense vector respond abnormally to ionizing radiation. Int J Mol Med 4: 43-47, 1999

6. Barzon L, Zanusso M, Colombo F and Palu G: Clinical trials of gene therapy, virotherapy, and immunotherapy for malignant gliomas. Cancer Gene Ther 13: 539-554, 2006.

7. Guha C, Guha U, Tribius S, et al: Antisense ATM gene therapy: a strategy to increase the radiosensitivity of human tumors. Gene Ther 7: 852-858, 2000.

8. Fan Z, Chakravarty P, Alfieri A, Pandita TK, Vikram B and Guha C: Adenovirus-mediated antisense ATM gene transfer sensitizes prostate cancer cells to radiation. Cancer Gene Ther 7: 1307-1314, 2000.

9. Pfeifer A and Verma IM: Gene therapy: promises and problems. Annu Rev Genomics Hum Genet 2: 177-211, 2001.

10. Dillon C: http://web.mit.edu/ccr/labs/jacks/protocols/rnairesources.htm. MIT Center For Cancer Research: Massachusetts, 2005.

11. Rubinson DA, Dillon CP, Kwiatkowski AV, et al: A lentivirusbased system to functionally silence genes in primary mammalian cells, stem cells and transgenic mice by RNA interference. Nat Genet 33: 401-406, 2003.

12. Sambrook J, Fritsch EF and Maniatis T: Molecular Cloning: A Laboratory Manual. Cold Spring Harbor Laboratory Press, New York, 1989 .

13. Gueven N, Becherel OJ, Howe O, et al: A novel form of ataxia oculomotor apraxia characterized by oxidative stress and apoptosis resistance. Cell Death Differ 14: 1149-1161, 2007.

14. Franken NA, Rodermond HM, Stap J, Haveman J and van Bree C: Clonogenic assay of cells in vitro. Nat Protoc 1: 23152319, 2006.
15. Morgan SE, Lovly C, Pandita TK, Shiloh Y and Kastan MB: Fragments of ATM which have dominant-negative or complementing activity. Mol Cell Biol 17: 2020-2029, 1997.

16. Bakkenist CJ and Kastan MB: DNA damage activates ATM through intermolecular autophosphorylation and dimer dissociation. Nature 421: 499-506, 2003.

17. Kozlov SV, Graham ME, Peng C, Chen P, Robinson PJ and Lavin MF: Involvement of novel autophosphorylation sites in ATM activation. EMBO J 25: 3504-3514, 2006

18. Beamish $\mathrm{H}$ and Lavin MF: Radiosensitivity in ataxia-telangiectasia: anomalies in radiation-induced cell cycle delay. Int J Radiat Biol 65: 175-184, 1994.

19. Kastan MB, Zhan Q, el-Deiry WS, et al: A mammalian cell cycle checkpoint pathway utilizing p53 and GADD45 is defective in ataxia-telangiectasia. Cell 71: 587-597, 1992.

20. Khanna KK and Lavin MF: Ionizing radiation and UV induction of p53 protein by different pathways in ataxia-telangiectasia cells. Oncogene 8: 3307-3312, 1993

21. Maser RS, Monsen KJ, Nelms BE and Petrini JH: hMrel1 and hRad50 nuclear foci are induced during the normal cellular response to DNA double-strand breaks. Mol Cell Biol 17: 6087-6096, 1997.

22. DeAngelis LM: Brain tumors. N Engl J Med 344: 114-123, 2001.

23. Stupp R, Mason WP, van den Bent MJ, et al: Radiotherapy plus concomitant and adjuvant temozolomide for glioblastoma. $\mathrm{N}$ Engl J Med 352: 987-996, 2005.

24. Breckpot K, Aerts JL and Thielemans K: Lentiviral vectors for cancer immunotherapy: transforming infectious particles into therapeutics. Gene Ther 14: 847-862, 2007.

25. Kafri T, Morgan D, Krahl T, Sarvetnick N, Sherman L and Verma I: Cellular immune response to adenoviral vector infected cells does not require de novo viral gene expression: implications for gene therapy. Proc Natl Acad Sci USA 95: 11377-11382, 1998.

26. Ginn S, Fleming J, Curtin J and Alexander I: Gene therapy: a roller-coaster ride. Today's Life Sci 15: 26-29, 2003.

27. Uziel T, Lerenthal Y, Moyal L, Andegeko Y, Mittelman L and Shiloh Y: Requirement of the MRN complex for ATM activation by DNA damage. EMBO J 22: 5612-5621, 2003.

28. You Z, Bailis JM, Johnson SA, Dilworth SM and Hunter T: Rapid activation of ATM on DNA flanking double-strand breaks. Nat Cell Biol 9: 1311-1318, 2007.

29. Berkovich E, Monnat RJ Jr and Kastan MB: Roles of ATM and NBS1 in chromatin structure modulation and DNA double-strand break repair. Nat Cell Biol 9: 683-690, 2007.

30. Kurz EU, Douglas P and Lees-Miller SP: Doxorubicin activates ATM-dependent phosphorylation of multiple downstream targets in part through the generation of reactive oxygen species. J Biol Chem 279: 53272-53281, 2004.

31. Shiloh Y: The ATM-mediated DNA-damage response: taking shape. Trends Biochem Sci 31: 402-410, 2006.

32. Lavin MF and Gueven N: The complexity of p53 stabilization and activation. Cell Death Differ 13: 941-950, 2006.

33. Steele RJ, Thompson AM, Hall PA and Lane DP: The p53 tumour suppressor gene. Br J Surg 85: 1460-1467, 1998.

34. Lavin MF and Shiloh Y: The genetic defect in ataxia-telangiectasia. Annu Rev Immunol 15: 177-202, 1997.

35. Hickson I, Zhao Y, Richardson CJ, et al: Identification and characterization of a novel and specific inhibitor of the ataxiatelangiectasia mutated kinase ATM. Cancer Res 64: 9152-9159, 2004.

36. Ivanov VN, Zhou H, Partridge MA and Hei TK: Inhibition of ataxia telangiectasia mutated kinase activity enhances TRAILmediated apoptosis in human melanoma cells. Cancer Res 69: 3510-3519, 2009. 